\title{
Vorstellungen von Lohngerechtigkeit in West- und Ostdeutschland und in der Ukraine
}

Unter welchen Umständen gilt ein bestimmtes Erwerbseinkommen als fair und gerecht? Geht es gerecht zu, wenn jede und jeder nach der erbrachten Leistung entlohnt wird oder nach seinem bzw. ihrem Bedarf? Gilt das als gerecht, was man gewöhnt ist? Oder unterstützen die Menschen Verteilungsprinzipien, bei denen sie selbst besser wegkommen als andere? Gerade in sich rasant verändernden Gesellschaften, in denen sich die Einkommensverteilung tief greifend wandelt, stehen Idealbilder von Gerechtigkeit in besonderer Weise auf dem Prüfstand. Die Vorstellungen von Einkommensgerechtigkeit in zwei Transformationsgesellschaften, Ostdeutschland und der Ukraine, sowie in der Referenzgesellschaft Westdeutschlands unterscheiden sich eindrücklich. ${ }^{\bullet}$

KATRIN AUSPURG, KSENIJA GATSKOVA, THOMAS HINZ

\section{Einleitung}

Die Ideen und Vorstellungen zu einer gerechten Verteilung von Ressourcen beeinflussen soziale Prozesse auf der individuellen sowie gesamtgesellschaftlichen Ebene (für einen Überblick siehe z. B. Liebig 1997). Mögliche Diskrepanzen zwischen den Gerechtigkeitseinstellungen verschiedener sozialer Gruppen erhöhen die Unzufriedenheit dieser Gruppen und in der Folge die Wahrscheinlichkeit der Mobilisierung zu sozialen Bewegungen (Lindenberg 1989; Opp 1988). Makrosoziologisch betrachtet steht das Gerechtigkeitsempfinden der Bevölkerung daher in einem direkten Zusammenhang mit der Legitimität politischer Institutionen, den Inhalten politischer Reformprozesse und sozialer Ordnung und Stabilität. Auf der individuellen Ebene gilt das Gerechtigkeitsempfinden in Bezug auf Entlohnungen als mitbestimmend für das Arbeitsklima in Betrieben, für die Arbeitsleistung und Aufstiegsmotivation, für Bildungsinvestitionen und auch für den Gesundheitszustand und die Lebenszufriedenheit von Menschen (Alwin 1987; Liebig et al. 2004).

Ziel der vorliegenden Untersuchung ist es, die Vorstellungen von gerechter Einkommensverteilung in drei unterschiedlichen gesellschaftlichen Umgebungen zu vergleichen und damit auch die Entstehung unterschiedlicher Verteilungsprinzipien zu erforschen. Verglichen werden eine entwickelte westeuropäische Demokratie (Westdeutschland), eine Transformationsgesellschaft mit vollzogener Transformation (Ostdeutschland) und eine post-sowjetische Transformationsgesellschaft (die Ukraine). Eine der wichtigsten Eigenheiten der Transformation in Mittel- und Osteuropa ist der Übergang von einer vormals lang anhaltenden und ideologisch gestützten Erfahrung relativer Gleichheit zu teils extrem ausgeprägter Ungleichheit, vor allem in der Einkommensverteilung. Der Vergleich dieser drei Bevölkerungen erscheint daher besonders geeignet, um herauszufinden, inwieweit die Befürwortung unterschiedlicher Verteilungsprinzipien eher durch eine Maximierung des eigenen Nutzens (Eigeninteresse) oder die Prägung und Anpassung an bestehende Verhältnisse bedingt ist.

Bislang vorliegende Studien zu Gerechtigkeitseinstellungen in kapitalistischen Staaten und den Transformationsländern wurden vor allem im Rahmen der ISJP-Erhebungen (International Social Justice Project) in den Jahren 1991, 1996 und - mit einem kleineren Kreis partizipierender Länder - in den Jahren 2000 und 2006 durchgeführt (siehe Kluegel et al. 1995 oder die Beiträge im Sonderheft des Social Justice Research 2/2000). Die Mehrzahl der Publikationen, die diese Datensätze verwenden, stellen fest, dass

Für hilfreiche Anmerkungen und Hinweise bedanken wir uns bei zwei anonymen Gutachtenden der WSIMitteilungen sowie bei Moritz Schirmböck und Andreas Schneck. 
die Transformationsgesellschaften zu einer etwas stärkeren Befürwortung von Egalität in der Einkommensverteilung neigen. Zunächst war eine Annäherung an die westlichen Gerechtigkeitsvorstellungen erkennbar. Nach anfänglicher Euphorie über die sozio-ökonomischen Entwicklungen zeigte sich bei späteren Untersuchungen dann aber eine zunehmende Skepsis gegenüber den neuen Marktgesetzen. Diese schlug sich in sinkendem Vertrauen in politische Institutionen und insbesondere bei der älteren Generation auch in größerem Fatalismus nieder (Wegener et al. 2000).

Ferner wurde in den vergleichenden Analysen - ausgehend von der theoretischen Unterscheidung zwischen den Gerechtigkeitsprinzipien Leistung, Bedarf und Gleichheit - in der Regel vermutet, dass die Bürger post-sowjetischer Länder deshalb in geringerem Maße Einkommensungleichheiten akzeptieren (Hadler 2005), weil sie stärker von dem in sozialistischer Ideologie verankerten Prinzip relativer Einkommensgleichheit ausgehen. Im Vergleich zu westlichen Ländern wird demnach dem Bedarfsprinzip ein gröBerer Stellenwert zugemessen (Marshall et al. 1999; für empirische Studien: Mason 1995; Wegener/Steinmann 1995). Entgegen den Erwartungen findet man jedoch keine klaren Ergebnisse dafür, dass dem Leistungsprinzip in den Transformationsgesellschaften eine geringere Bedeutung zukommt (Cohn et al. 2000). Angesichts dieser Ergebnisse stellt sich die Frage, ob die gleichzeitige Relevanz sehr unterschiedlicher Prinzipien nicht auch ein Artefakt der Umfrageforschung darstellt: Möglicherweise hat die bislang vorherrschende sukzessive Abfrage von einzelnen Verteilungsprinzipien den Befragten zu wenig Abwägungsprozesse abverlangt (siehe z. B. Arts et al. 1991 für eine kritische Bestandsaufnahme der empirischen Gerechtigkeitsforschung).

Ein weithin konformes Ergebnis der Vergleichsstudien besagt aber, dass Einkommensgerechtigkeit in den Transformationsgesellschaften inkonsistenter beurteilt wird als in den westlichen Ländern (z. B. Wegener/Liebig 1995; Wegener/Steinmann 1995). Hierfür gibt es unterschiedliche Erklärungen: Zum einen wird vermutet, dass inkonsistente Gerechtigkeitseinstellungen durch ein Aufeinandertreffen alter und neuer Normen oder Sozialisationsmuster hervorgerufen werden (Mason 1995). Zum anderen können die Befragten unschlüssig darüber bleiben, welche Prinzipien sie rational verfolgen sollten, da die Entlohnungsprinzipien und ihre eigene Position im sozialen Gefüge der Transformationsgesellschaften noch zu wenig etabliert sind (Liebig 1997, S. 172ff.). Schließlich wird davon ausgegangen, dass Gerechtigkeitseinstellungen auch stark durch die bestehenden Verhältnisse geprägt werden. In Transformationsländern mit unklaren Entlohnungsprinzipien ist dann entsprechend auch die „normative Kraft des Faktischen“ noch schwach entfaltet (Kluegel et al. 1995).

\section{Theorie und Forschungshypothesen}

Die theoretische Diskussion zu Gerechtigkeitsvorstellungen führt zu der Frage, wie Ideen von gerechten Verteilungsprinzipien, im vorliegenden Fall: von gerecht empfundenen Entlohnungen für Erwerbsarbeit, in den jeweiligen Bevölkerungen zustande kommen. Die Diskussion ist an dieser Stelle bewusst verengt; weitestgehend ausgeschlossen werden philosophische Begründungszusammenhänge und politische Meinungsbildungen. Abstrahiert man hiervon, erscheinen Gerechtigkeitseinstellungen zum einen durch kulturelle und normative Rahmenbedingungen geprägt. Dieser Theoriestrang lässt sich nochmals in Prozesse der Sozialisation und der Adaption an vorliegende Bedingungen untergliedern. Zum anderen dürften sich Gerechtigkeitseinstellungen an dem Eigeninteresse der einzelnen Akteure orientieren, was eine mit der sozialen Position variierende Befürwortung von Verteilungsregeln impliziert. ${ }^{2}$ Unklar ist das Gewicht der einzelnen Faktoren, weshalb sich die nachfolgende Ableitung von Hypothesen auf eine Trennung der verschiedenen Mechanismen konzentriert.

\subsection{Sozialisation und Lernen}

Kulturelle Werte und damit einhergehende Normen und Einstellungen werden nach Sozialisations- und Lerntheorien insbesondere während des Heranwachsens in der Familie oder in Bildungsinstitutionen internalisiert. Gerechtigkeitsvorstellungen sind demzufolge nicht nur eine Frage individueller Standpunkte (,justice is in the eye of the beholder" [Walster et al. 1973]), sondern sie sind auch wesentlich durch historische Zeitpunkte, politische Systeme und die dort vorherrschenden Ideologien geprägt. Weltanschauliche Grundsätze, allgemeine Wertbindungen und politische Einstellungen werden vor dem Hintergrund des jeweiligen kulturellen Kontextes von Gesellschaften vor allem in der frühen Adoleszenz geformt und erweisen sich dann für die späteren Lebensphasen als sehr stabil (Alwin/ Krosnick 1991). Die in den frühen Lebensphasen internalisierten Einstellungen weisen somit eine lebenslange Prägekraft auf (Wegener et al. 2000; Wegener/Liebig 1993, S. 667). Dies gilt auch aus entwicklungspsychologischer Sicht, der zufolge verinnerlichte Normen Teil der Person sind, eine Facette ihres Selbst oder ihrer Identität bilden (Oerter/Montada 1998, S. 866).

(2) Zumindest die Adaption lässt starke Übereinstimmungen innerhalb von Gesellschaften erwarten, in stabilen Gesellschaften gilt dies ebenso für die Sozialisation. Für derartige allgemein geteilte Gerechtigkeitsvorstellungen wird auch der Begriff „primärer Ideologien“ verwendet, während nur in Teilgruppen vorherrschende Einstellungen als "sekundäre Ideologien“ bezeichnet werden (Liebig/Wegener 1995). 
Dies legt für die vorliegende vergleichende Studie zunächst die Vermutung nahe, dass grundsätzliche Länderunterschiede bestehen. Unsere erste Hypothese $(H)$ lautet daher: Im westlichen Vergleichsland wird die Entlohnung nach Leistung generell stärker befürwortet als in den Transformationsgesellschaften $\left(H_{1 a}\right)$. Dies bedingt sich durch eine im Westen ausgeprägtere Sozialisation in Normen und Werte des Liberalismus und Individualismus (Almond/Verba 1963; für jüngere Untersuchungen siehe insbesondere die Vergleichsstudie zu osteuropäischen Transformationsgesellschaften von Pickel et al. 2006). Weiter sollte nach der Sozialisationsthese in den Transformationsländern, anders als in westlichen Ländern, ein Generationenunterschied bestehen. Die jüngere Generation, die sich über fehlende Arbeitserfahrung vor der Transformationsphase (also die Zeitspanne 1989 - 1991) definieren lässt, machte die Berufssozialisation bereits in einem neuen System der Arbeitsorganisation durch. Dieses war nicht mehr von Staatseigentum und Lenkung der Arbeitskräfte geprägt, sondern stärker durch Angebot und Nachfrage auf dem Arbeitsmarkt und durch die Betonung individueller Leistung. Im Gegensatz dazu wurde die Sozialisation der älteren Generation von sowjetischer Ideologie und planwirtschaftlicher Entlohnungspraxis, die keine starken Lohnungleichheiten zuließen, beeinflusst. Entsprechend sollte die jüngere Generation in den Transformationsgesellschaften eine erheblich größere Einkommensungleichheit akzeptieren als die ältere, was zu der These führt: Insbesondere in den Transformationsgesellschaften befürwortet die ältere Generation die Prinzipien Bedarf und Gleichheit stärker als die jüngere Generation $\left(H_{1 b}\right)$.

\subsection{Adaption}

Ein weiterer Mechanismus, der den prägenden Einfluss gesellschaftlicher und historischer Kontexte auf Gerechtigkeitseinstellungen betont, wird als normative Kraft des Faktischen bezeichnet. Dieser Ausdruck hebt darauf ab, dass bestehende Verhältnisse wesentliche Grundlagen für die Prägung normativer Einstellungen bilden, wie es George $C$. Homans zum Ausdruck gebracht hat: „What is determines what always ought to be. It is this actual, common experience that provides the basis for the general rule of justice" (Homans 1973, S. 579; Hervorhebungen im Original). Dieser Adaptionsthese zufolge sollten nach einer kurzen Phase der Anpassung die Gerechtigkeitsurteile den Standards des (gerade) Üblichen bzw. den wahrgenommenen Entlohnungsprinzipien entsprechen. Dazu nochmals Homans: „ [...] what people say ought to be is determined in the long run and with some time lag by what they find in fact to be the case" (Homans 1974, S. 250). Eine sozialpsychologische Begründung für diese These wird durch die Theorie kognitiver Dissonanz geliefert, welche besagt, dass Menschen dazu neigen, einander widersprechende Wissenselemente, Überzeugungen und Erfahrungen miteinander in Einklang zu bringen (Festinger 1957). Gerade angesichts schwer überwindbarer Missstände kann die Umdeutungsleistung wich- tig werden, um das psychische Gleichgewicht zu bewahren. Statt wenig aussichtsreicher Veränderung der Verhältnisse wird dann eine Uminterpretation von Einstellungen und Normen vorgenommen (ähnliche Annahmen wurden auch in Anomie-Theorien formuliert, siehe dazu Merton 1938, 1957). Nach der Adaptionsthese ist jedenfalls zu erwarten, dass länderspezifische Unterschiede in den Gerechtigkeitseinstellungen sich analog zu den (wahrgenommenen) tatsächlichen Entlohnungsprinzipien zeigen $\left(\mathrm{H}_{2 a}\right)$. Aufgrund der empirisch zu beobachtenden zeitlichen Verzögerung von Adaptionen nach Transformationen (Kluegel et al. 1995; Wegener/Steinmann 1995) und der höheren Stabilität von Entlohnungsprinzipien in West- und Ostdeutschland sollte diese Adaption allerdings etwas stärker in West- und Ostdeutschland als in der Ukraine zu beobachten sein $\left(H_{2 b}\right)$.

\subsection{Eigeninteresse und Rational Choice}

Nach Rational Choice Theorien werden Personen nicht nur passiv durch Normen und Einstellungen beeinflusst, sondern sie wirken selbst auch aktiv - und orientiert an einer Maximierung ihres eigenen Wohlbefindens - an der Aufstellung und Durchsetzung von Normen mit (Coleman 1990; Opp 1983). Demnach ist zu erwarten, dass die von den Akteuren befürworteten Prinzipien gerechter Entlohnung mit ihren persönlichen Verteilungsinteressen und in der Folge ihrer sozialen Position variieren (Liebig 1997). Es werden primär solche Prinzipien unterstützt, die den eigenen materiellen Interessen dienen. Somit sollten sich innerhalb der Gesellschaften Unterschiede in den Gerechtigkeitseinstellungen finden, die vor allem anhand von Einkommens- und Bildungsunterschieden markiert werden. Personen mit höherem Einkommens- und Bildungsniveau sollten stärker als andere Schichten Bezahlungen nach dem Leistungsprinzip befürworten und Umverteilungen ablehnen. Im vorliegenden Aufsatz soll diese Eigeninteresse-These beispielhaft anhand der Annahme geprüft werden, dass in allen untersuchten Ländern analoge Gruppenunterschiede dahin gehend bestehen, dass besser gebildete Personen die Entlohnung nach Leistung stärker befürworten als gering gebildete $\left(\mathrm{H}_{3 a}\right)$. Ferner ist davon auszugehen, dass eine klare und konsistente Normenunterstützung im Sinne des Eigeninteresses vor allem in Gesellschaften zu beobachten ist, in denen die Entlohnungsprinzipien bereits stabil verankert sind: In Westdeutschland variieren die Gerechtigkeitseinstellungen stärker mit dem Bildungsniveau als in den Transformationsgesellschaften $\left(H_{3 b}\right)$.

\subsection{Hypothesen-Zusammenfassung}

Die genannten Prinzipien schließen sich nicht unbedingt aus, sondern es ist nach vorliegendem Wissensstand eher von einer gegenseitigen Ergänzung auszugehen: Rationales Handeln erfolgt vor dem Hintergrund des politischen und normativen Kontextes und beeinflusst im Gegenzug die Normsetzung in Gesellschaften. Zugleich helfen Ad- 
aptionen an bestehende Praktiken, Dissonanzgefühle zu reduzieren, sie überschreiben aber nicht vollständig die Prägung durch frühe Sozialisationserfahrungen. Gleichwohl lassen die Theorien ein unterschiedliches Gewicht der einzelnen Prinzipien in den drei Länderkontexten erwarten, was die empirische Trennung der einzelnen Mechanismen sehr interessant macht. So sind etwa nach der Sozialisationsthese in den Transformationsländern stärker ausgeprägte Altersunterschiede zu vermuten als im westlichen Vergleichsland, während nach der Eigeninteresse-Hypothese insbesondere in Westdeutschland Einstellungsunterschiede nach Bildungshintergrund zu erwarten sind. Nach der Adaptionsthese spiegeln in allen und vor allem in stabilen Gesellschaften die vorherrschenden Gerechtigkeitseinstellungen überdies die realen Verteilungsprinzipien wider.

\section{Methodik und Datengrundlage}

Frühere Studien konzentrieren sich, sofern überhaupt Gerechtigkeitseinstellungen gemessen werden, fast alle auf sehr abstrakte Prinzipien. Etwa wird in Umfragen danach gefragt,

\section{ÜBERSICHT 1}

\section{Beispielvignette für Gerechtigkeitsbewertungen*}

Eine 45-jährige, verheiratete Frau mit zwei Kindern, deren Mann kein eigenes Einkommen bezieht, hat eine abgeschlossene Berufsausbildung und arbeitet derzeit als Friseurin in einem Großbetrieb, der kurz vor dem Konkurs steht.

Sie erbringt an ihrem Arbeitsplatz eher unterdurchschnittliche Leistungen und verdient im Monat 1200 Euro brutto, d. h. vor Abzug von Steuern und Abgaben.

\section{Ihre Bewertung:}

F $1:$ Ist das monatliche Brutto-Einkommen für diese Person Ihrer Meinung nach gerecht oder ungerecht?

$\square$ Einkommen ist gerecht $(\rightarrow$ Weiter mit der nächsten Personenbeschreibung)

$\square$ Einkommen ist ungerecht $(\rightarrow$ Weiter mit F 2)

F 2:

Ist das Einkommen ungerechterweise zu hoch oder zu niedrig?

$\square$ Ungerechterweise zu hoch ( $\rightarrow$ Weiter mit F 3)

$\square$ Ungerechterweise zu niedrig ( $\rightarrow$ Weiter mit F 3)

F 3:

Welche Zahl zwischen 1 und 100 drückt das Ausmaß an Ungerechtigkeit, das Sie persönlich empfinden, am besten aus? ob Einkommensunterschiede zu groß sind oder ob Einkommen vor allem nach Leistung oder Bedarf verteilt werden sollte (siehe etwa die Beiträge in Kluegel et al. 1995). Die Abfrage derart abstrakter Prinzipien führt womöglich zu einer Unterschätzung tatsächlich bestehender Einstellungsunterschiede (Miller 2008, S. 127f.).

Alternativ kommt in der vorliegenden Untersuchung eine Methode zum Einsatz, die bereits in früheren Arbeiten zur Aufdeckung der normativen Struktur von Gerechtigkeitsurteilen verwendet wurde (z. B. Auspurg et al. 2008; Alves/Rossi 1978; Jasso/Rossi 1977; Jasso/Webster 1999). Es handelt sich um das Faktorielle Survey (FS) Design. Die Besonderheit der Methodik besteht darin, dass den Befragten mehrere fiktive Beschreibungen von Erwerbspersonen (Vignetten) vorgelegt werden; die beschriebenen Situationen sollen sie im Hinblick auf deren Gerechtigkeit bewerten (vgl. hierzu grundlegend: Beck/Opp 2001; Jasso 2006; Rossi/Nock 1982). In den Beschreibungen werden einzelne Merkmale experimentell variiert, was es erlaubt, ihren Einfluss auf die Bewertungen festzustellen. Im vorliegenden Fall bewerten die Befragten die Gerechtigkeit der BruttoLohnhöhe von fiktiven Erwerbstätigen, die sich in Merkmalen wie Geschlecht, Ausbildung, Beruf oder gezeigter Arbeitsleistung unterscheiden (siehe Übersicht 1).

Die in den folgenden Analysen benutzten FS-Daten stammen aus zwei Befragungen: Dem Pretest des Soziooekonomischen Panels (SOEP) aus dem Jahr 2008 für Westund Ostdeutschland (Sauer et al. 2009) und dem Omnibus des Jahres 2009 der Ukraine (Institute of Sociology of the National Academy of Sciences of Ukraine 2009). Die Befragung des SOEP-Pretests wurde in Form von Computer Assisted Personal Interviews (CAPI) im August 2008 durchgeführt. ${ }^{8}$ Insgesamt nahmen 1.066 Personen der deutschen Wohnbevölkerung im Alter ab 16 Jahren an der Befragung teil. Für die nachfolgende Analyse werden nur die 944 Befragten berücksichtigt, die bereits vor der Wende geboren wurden und auch nicht im Ausland gelebt haben (davon ca. 80 \% mit Wohnsitz in Westdeutschland und $20 \%$ mit Wohnsitz in Ostdeutschland). Der Fragebogen enthielt ein FS-Modul, in welchem den Befragten jeweils 24 Fallbeschreibungen fiktiver Einkommensbezieher vorgelegt wurden. Insgesamt kamen 240 unterschiedliche Fallbeispiele zum Einsatz, jedem Befragten wurde zufällig eine von zehn unterschiedlichen Fragebogenversionen (Decks) zugewiesen (zu weiteren Details: Sauer et al. 2009).

Die Daten für die ukrainische Bevölkerung wurden im Rahmen von Omnibus 2009 erhoben. Das FS-Modul wurde weitgehend parallel angelegt, setzte sich aufgrund der geringeren verfügbaren Befragungszeit aber nur aus jeweils zwölf Vignetten pro Befragten zusammen. 240 Vignetten wurden auf 20 unterschiedliche Decks aufgeteilt (gebildet 


\section{Vignettendimensionen und ihre Ausprägungen}

\begin{tabular}{|c|c|c|}
\hline \multirow{3}{*}{$\begin{array}{l}\text { Dimensionen } \\
\text { Alter }\end{array}$} & \multicolumn{2}{|c|}{ Ausprägungen } \\
\hline & Deutschland & Ukraine* \\
\hline & $25,35,45,55$ & $25,35,40,50$ \\
\hline Geschlecht & \multicolumn{2}{|l|}{ Männlich, weiblich } \\
\hline Familienstand & \multicolumn{2}{|c|}{ Alleinverdiener verheiratet, Zweiverdiener verheiratet, alleinstehend } \\
\hline Ausbildungsabschluss & \multicolumn{2}{|c|}{ Ohne Berufsabschluss, mit abgeschlossener Berufsausbildung, mit Hochschulabschluss } \\
\hline Beruf (nach Prestige geordnet) ${ }^{* *}$ & $\begin{array}{l}\text { Hilfsarbeiter(in), Pförtner(in), Lokführer(in), } \\
\text { Verwaltungsfachkraft, Friseur(in), Sozialarbeiter(in), } \\
\text { Programmierer(in), Elektroingenieur(in), } \\
\text { Leitende(r) Manager(in), Arzt (Ärztin) }\end{array}$ & $\begin{array}{l}\text { Kellner(in), Kaufmann (Kauffrau), Friseur(in), } \\
\text { Bauarbeiter(in), Buchhalter(in), Arzt (Ärztin), } \\
\text { Journalist(in), Universitätsprofessor(in), } \\
\text { Unternehmer(in), Anwalt (Anwältin) }\end{array}$ \\
\hline Einkommen (brutto) in EUR bzw. UAH & $500,950,1200,1500,2500,3800,5400,6800,10000,15000$ & $400,600,800,1200,1750,3000,4000,5000,8000,15000$ \\
\hline Kinder & \multicolumn{2}{|l|}{ Kein Kind, 1 Kind, 2 Kinder, 3 Kinder, 4 Kinder } \\
\hline Leistung am Arbeitsplatz & \multicolumn{2}{|l|}{ Unter-, über-, durchschnittlich } \\
\hline Betriebssituation & \multicolumn{2}{|c|}{ Hohe Gewinne, vom Konkurs bedroht, wirtschaftlich stabil } \\
\hline Betriebsart & Groß-, Mittel-, Kleinbetrieb & Privat-, Staatsbetrieb \\
\hline \multicolumn{3}{|c|}{$\begin{array}{l}\text { *Für die Ukraine kann der bislang unveröffentlichte Fragebogen bei den Autoren angefordert werden. Die Abweichungen in den Ausprägungen zwischen Deutschland und der Ukraine } \\
\text { begründen sich durch bessere Anpassungen an die jeweiligen Arbeitsmärkte, so folgt etwa das Spektrum der ausgewählten Berufe jeweils den länderspezifischen Prestigeverteilungen } \\
\text { (Auswahl von jeweils einem Beruf aus jedem Dezentil). } \\
\text { **In den Auswertungen wird statt dem Beruf das Berufsprestige nach länderspezifischen Prestigeskalen eingesetzt: für Deutschland die Magnitude-Prestigeskala (Christoph 2005), } \\
\text { ebenso wurde für die Ukraine eine einschlägige Skala eingesetzt (Oksamytna/Patrakova 2007). }\end{array}$} \\
\hline Ile: Sauer et al. (2009). & & \\
\hline
\end{tabular}

aus derselben Vignettenstichprobe wie im SOEP-Pretest, um die Vergleichbarkeit zu gewährleisten). Der Fragebogen wurde von Interviewern zur Selbstausfüllung verteilt und von 1.797 Personen im Alter ab 18 Jahren bearbeitet. Die Befragtenstichprobe gilt als repräsentativ für die erwachsene Bevölkerung der Ukraine.

Eine Beispielvignette, wie sie in Deutschland - und mit kleinen Anpassungen an die grammatikalische Sprachlogik und die realen Arbeitsmarktstrukturen in der Ukraine verwendet wurde, ist in Übersicht 1 dargestellt.

Die in Übersicht 1 kursiv hervorgehobenen Wörter zeigen die Merkmale an, die über die 240 Fallbeispiele hinweg variiert wurden. Viele dieser Merkmale stehen explizit für ein bestimmtes Gerechtigkeitsprinzip: Das Leistungsprinzip wird beispielsweise anhand der gezeigten Arbeitsleistung und Ausbildung der Vignettenperson vorgegeben. Das Bedarfsprinzip wird durch die Anzahl der Kinder, den Familienstand (d. h. ob es einen weiteren Verdiener im Haushalt gibt) und das Alter operationalisiert. Die Einschätzung der in den Vignetten vorgegebenen Einkommensspanne erlaubt zudem eine Abschätzung dazu, inwieweit Ungleichheiten toleriert werden oder das Gleichheitsprinzip befürwortet wird. Die Variablen Berufsprestige und Geschlecht weisen auf die mögliche zusätzliche Rolle von Status bzw. von Prinzipien der Askription hin (Ridgeway 1991). Die Studie konzentriert sich bewusst auf das Bruttogehalt, das Arbeitgeber ihren Beschäftigten bezahlen - und nicht auf die Vorstellungen von einem gerechten Einkommen nach der Umverteilung durch Steuern. Dies erlaubt es besonders gut, die
Entlohnungspraktiken nach der Marktlogik zu betrachten. Die vollständige Liste der in den Vignetten enthaltenen Merkmale und ihren Ausprägungen ist in Übersicht 2 aufgeführt. Die Befragten sollten für alle Fallbeispiele jeweils angeben, ob das genannte Bruttoeinkommen für die geschilderte Person angemessen, zu hoch oder zu niedrig ist (siehe ebenfalls Übersicht 1). ${ }^{\circledR}$

Zur Prüfung der Hypothesen $\mathrm{H}_{1 \mathrm{~b}}$ und $\mathrm{H}_{3}$ werden jeweils Teilgruppen der Befragten miteinander verglichen. Im Hinblick auf Kohortenunterschiede $\left(\mathrm{H}_{1 \mathrm{~b}}\right)$ werden Personen, die zum Zeitpunkt der politischen Wende (1989 in Deutschland bzw. 1991 in der Ukraine) maximal 30 Jahre alt waren, mit jenen verglichen, die älter waren. Im Alter von 30 Jahren sollte die Sozialisation genereller Werte und Normen weitgehend abgeschlossen sein. Zur Unterscheidung von Personen mit hohen und geringeren Einkommenschancen aufgrund eigener Leistung $\left(\mathrm{H}_{3}\right)$ werden Personen mit

(4) In der Ukraine wurde eine leicht abgewandelte Skala eingesetzt. Es stellte sich heraus, dass die an Magnitude-Skalierungen angelehnte Feinabstufung im SOEP-Pretest von den Befragten kaum ausgeschöpft wurde (Häufung auf gerundeten Angaben wie Vielfachem vom Wert Zehn). Im Omnibus in der Ukraine wurde daher auf eine zeiteffizientere elfstufige Ratingskala (von $-5=$ „ungerechterweise zu niedrig" über null $={ }_{\text {"gerecht }}$ " bis $5=$ "ungerechterweise zu hoch“) zurückgegriffen. Diese unterschiedlichen Antwortmessungen wurden jeweils anhand der Standardabweichungen normiert (z-Standardisierung, vgl. Jann 2005). 
ABB.

\section{Gerechtigkeitsurteile* im Vergleich}

semipartielle $R^{2}$-Werte

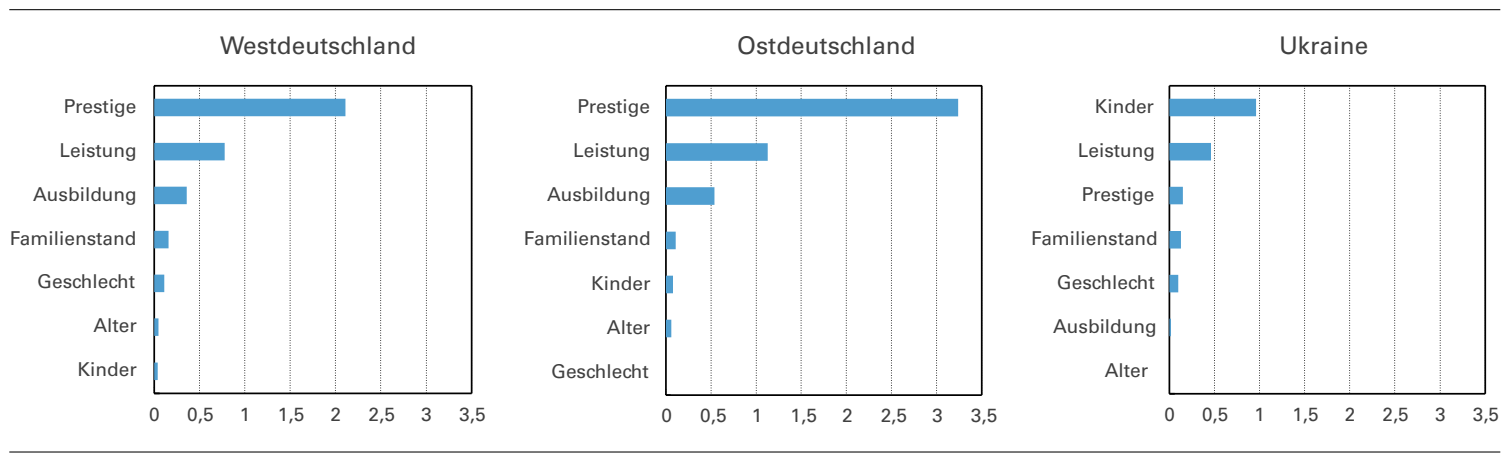

${ }^{*} \mathrm{~N}=17.688$ Vignettenurteile (737 Befragte) für Westdeutschland, $\mathrm{N}=4.968$ Vignettenurteile (207 Befragte) für Ostdeutschland, $\mathrm{N}=21.549$ Vignettenurteile (1.797 Befragte) für die Urkaine.

Quelle: SOEP Pretest (2008) bzw. Omnibus (2009); Berechnungen der Autoren.

Hochschulabschluss (in West- und Ostdeutschland) bzw. dem Abschluss einer beruflichen Ausbildung oder Hochschulbildung (Ukraine) den anderen Befragten gegenübergestellt. $^{5}$

Es werden im Folgenden Ordinary Least Square (OLS)Regressionen mit robusten Standardfehlern geschätzt ( $\mathrm{Hu}-$ ber-White-Korrekturen für die geclusterte Datenstruktur, die sich durch die mehrfache Beurteilungsaufgabe durch einzelne Befragte ergibt; siehe dazu Rogers 1993). Abhängige Variable ist jeweils das z-standardisierte Urteil der Gerechtigkeitseinschätzung (Standardisierung pro Befragtem, um die unterschiedliche Expressivität in Gerechtigkeitsurteilen zu berücksichtigen, dazu Jasso 2007). Als unabhängige Variable werden die Vignettenvariablen eingesetzt. Zur Beurteilung der relativen Wichtigkeit von Vignettendimensionen finden semipartielle $R^{2}$-Werte Verwendung (Soofi et al. 2000). ${ }^{\odot}$ Schließlich werden zur Einschätzung der Präferenz für Gleichheit auch die Spannweiten der als gerecht bewerteten Einkommen, etwa nach Geschlecht der Vignettenperson, berechnet. Hierbei kommt mit einer geringen Modifikation die sogenannte Jasso-Formel (2007) zum Einsatz, mit der sich die als gerecht empfundenen Lohnabstände indirekt aus den Urteilen erschließen lassen. ${ }^{\circledR}$ Unterschiede zwischen Befragtengruppen werden anhand von Interaktionstermen auf statistische Signifikanz getestet (Chow-Tests, vgl. Wooldridge 2006).

\section{Ergebnisse}

Die Vorstellung der Ergebnisse erfolgt in drei Schritten: Zunächst (Abschnitt 4.1) beziehen sich Regressionsanalysen der Vignettenurteile auf die allgemeinen Gerechtigkeitsprinzipien im Ländervergleich $\left(\mathrm{H}_{1 \mathrm{a}}\right)$. Anschließend (4.2) werden Gruppenunterschiede zwischen junger und alter Generation und zwischen hoch und niedrig Gebildeten thematisiert $\left(\mathrm{H}_{1 \mathrm{~b}}, \mathrm{H}_{3 \mathrm{a}}, \mathrm{H}_{3 \mathrm{~b}}\right)$. Schließlich (4.3) werden die in den jeweiligen tatsächlichen Arbeitsmärkten bestehenden Einflussfaktoren auf die Lohnbildung mit den Gerechtigkeitsurteilen verglichen. Dies gibt Hinweise auf das Ausmaß der Adaption $\left(\mathrm{H}_{2}\right)$.

\subsection{Relevanz der Gerechtigkeitsprinzipien im Ländervergleich $\left(\mathrm{H}_{1 \mathrm{a}}\right)$}

Der relative Einfluss der einzelnen Merkmale der Vignettenpersonen für die drei Umgebungen wird jeweils grafisch aufbereitet und in Rangreihen von semipartiellen $R^{2}$-Werten dargestellt. ${ }^{\circledR}$ Für Westdeutschland ergibt sich eine starke Orientierung der Lohngerechtigkeit am Berufsprestige ( $A b$ -

5 Die Berufsausbildung besitzt in der Ukraine einen vergleichsweise höheren Stellenwert, weshalb diese Art der Kodierung für einen internationalen Vergleich am sinnvollsten erschien.

6 Ein Nachteil dieses Maßes ist, dass die relative Wichtigkeit sich auch nach den Interkorrelationen der Variablen und der Reihenfolge bestimmt, mit der die Variablen in das Modell eingehen. Dies trifft allerdings kaum auf die verwendeten Vignettenstichproben zu, denn aufgrund des experimentellen Designs sind die einzelnen Merkmale kaum korreliert (für Details siehe wiederum Sauer et al. 2009).

(7) Die Modifikation besteht darin, a) nicht nur das Vignetteneinkommen, sondern auch alle anderen Vignettenvariablen bei der Schätzung zu berücksichtigen, und b) Befragtenübergreifende Schätzungen vorzunehmen, die sich als präziser erweisen (Hox et al. 1991; für Details zur hier verwendeten Formel: Auspurg/Jäckle 2012).

8 Die zugrunde liegenden Regressionstabellen können bei den Autoren angefordert werden. 
bildung 1). An zweiter Stelle steht die erbrachte Leistung am Arbeitsplatz und an dritter Stelle die Ausbildung. Beides sind Indikatoren für das Leistungsprinzip. Deutlich weniger einflussreich sind Familienstand, Geschlecht, Alter und die Zahl der Kinder - dies sind vornehmlich Merkmale, die für das Bedarfsprinzip stehen. Der starke Einfluss des Berufsprestigewerts in Westdeutschland weist auf eine offenbar hohe Akzeptanz von Einkommensunterschieden zwischen einzelnen Berufen hin (und damit auf eine starke normative Kraft des Faktischen, denn es wird auch real stark nach Berufen entlohnt; siehe etwa Statistisches Bundesamt 2009).

In Ostdeutschland sieht die Rangreihe beinahe identisch aus. Berufsprestige und die Leistung am Arbeitsplatz spielen sogar eine noch größere Rolle als in Westdeutschland. Einzig das Geschlecht steht an anderer Stelle in der Rangreihe. In Ostdeutschland ist es für die Einschätzung der gerechten Einkommen völlig unbedeutend, die Anzahl der Kinder ist hingegen etwas wichtiger als in Westdeutschland. Der tatsächliche Lohnunterschied zwischen den Geschlechtern ist und war in Ostdeutschland übrigens auch deutlich geringer als in Westdeutschland (Bispinck et al. 2008), was für die Adaptions- oder Sozialisationsthese spricht. Die Unterschiede zwischen West- und Ostdeutschland sind für die Aspekte Prestige und Geschlecht statistisch signifikant.

Die Ukraine gibt ein völlig anderes Bild ab: Hier ist die Anzahl der Kinder die bedeutendste Einflussgröße. Demnach spielen in der post-sowjetischen Transformationsgesellschaft Bedarfskriterien für Gerechtigkeitseinschätzungen eine sehr viel größere Rolle. Erwähnenswert ist jedoch auch, dass an zweiter Stelle bereits die erbrachte Leistung am Arbeitsplatz steht (vgl. auch Marshall et al. 1999). Die Ausbildung und das Berufsprestige sind hingegen vergleichsweise bedeutungslos. Die erste Sozialisationshypothese $\left(\mathrm{H}_{1 \mathrm{a}}\right)$, wonach in Transformationsgesellschaften das Leistungsprinzip in geringerem Ausmaß als Gerechtigkeitsprinzip verankert ist, kann somit nicht bestätigt werden. In der Ukraine ist aber die Bedarfsgerechtigkeit weit mehr akzeptiert als in den beiden Teilen Deutschlands. Der Vergleich der Spannweiten von gerechten Einkommen zeigt keine auffälligen Differenzen, in der Ukraine fällt der Quartilsabstand etwas kleiner aus als in West- und Ostdeutschland.

\subsection{Unterschiede zwischen Befragtengruppen $\left(\mathbf{H}_{1 b}, \mathbf{H}_{1 c}, \mathbf{H}_{3 a}, \mathbf{H}_{3 b}\right)$}

Die zweite Sozialisationshypothese $\left(\mathrm{H}_{1 \mathrm{~b}}\right)$ besagt, dass in Ostdeutschland und vor allem der Ukraine deutlichere Unterschiede zwischen der älteren und jüngeren Generation bestehen als in Westdeutschland. Insbesondere die älteren Befragten sollten in den erstgenannten Länderkontexten in ihrem Urteil die althergebrachte Orientierung am Bedarfsprinzip widerspiegeln, während die jüngere Generation in stärkerem Maße die erbrachte Leistung honoriert.

In Abbildung 2 sind jeweils signifikante Gruppenunterschiede (blaue Balken) innerhalb der Länder markiert. In Westdeutschland zeigt sich eine weitgehende Übereinstim-
ABB. 2

\section{Gerechtigkeitsurteile nach Kohorten im Vergleich*}

semipartielle $R^{2}$-Werte

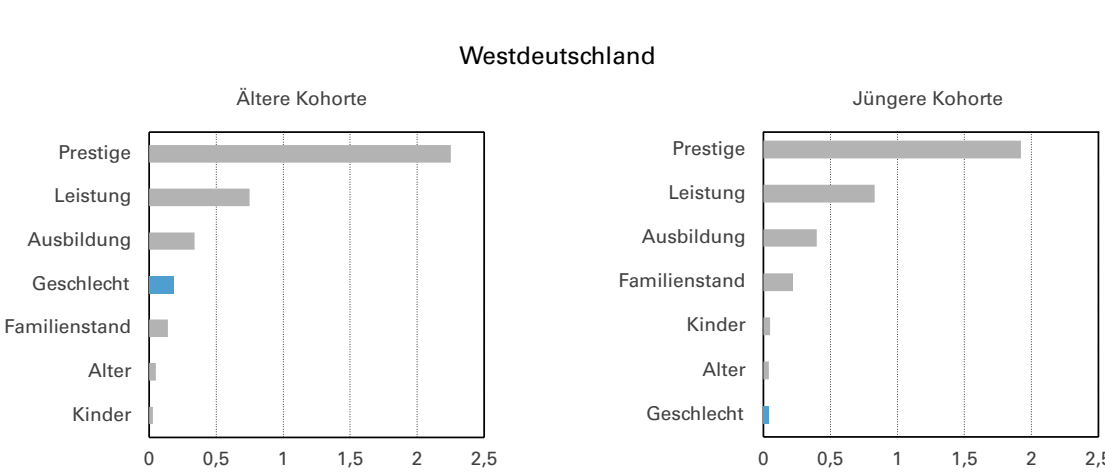

Ostdeutschland
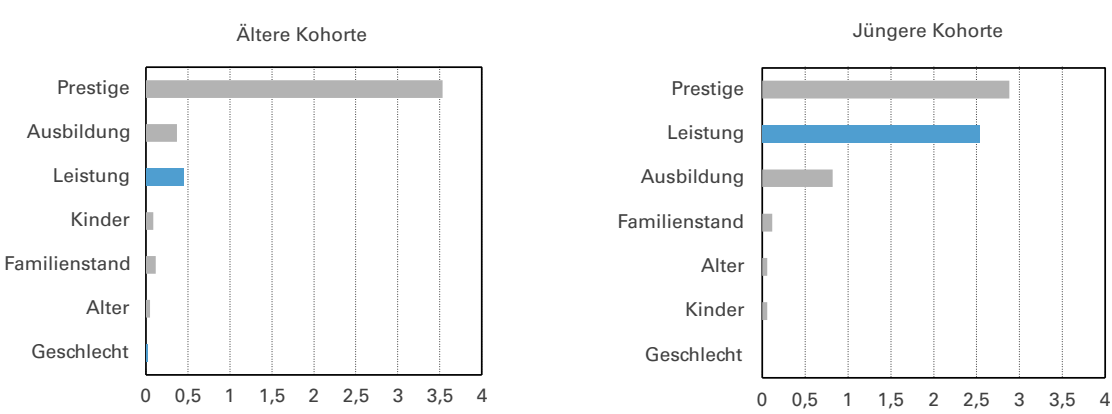

Ukraine
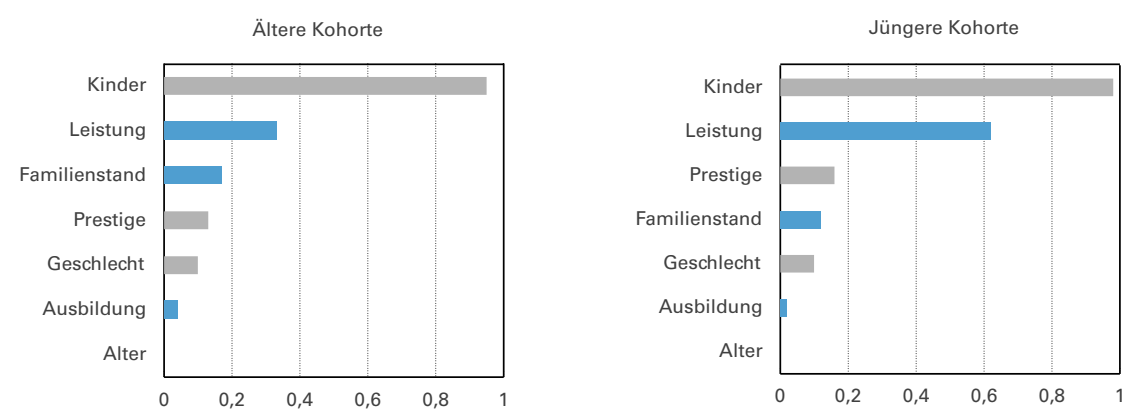

${ }^{*} \mathrm{~N}=10.656$ und 7.032 Vignettenurteile für die ältere und jüngere Kohorte in Westdeutschland (444 und 293 Befragte); 2.880 und 2.088 Vignettenurteile (120 und 87 Befragte) für Ostdeutschland; $N=9.719$ und 11.818 Vignettenurteile für die ältere und jüngere Kohorte in der Ukraine ( 811 bzw. 985 Befragte). Für eine bessere Lesbarkeit sind die $x$-Achsen länderspezifisch formatiert; die Werte sind abhängig von Fallzahlen und der generellen Varianzaufklärung.

Quelle: SOEP Pretest (2008) bzw. Omnibus (2009); Berechnungen der Autoren.

mung über Generationsgrenzen hinweg, einzig das Geschlecht wird in der jüngeren Generation als Merkmal nicht mehr unterstützt, während bei älteren Befragten noch traditionelle Rollenmuster verbreitet sind. In Ostdeutschland und der Ukraine zeigen sich in spezifischer Weise jeweils deutlichere Unterschiede im Generationenvergleich. In Ostdeutschland weichen die Urteile der Kohorten hinsichtlich eines Merkmals ab: Leistung am Arbeitsplatz ist für die jüngeren Befragten dort bedeutsamer für die Einkommensgerechtigkeit, was der Prognose von $\mathrm{H}_{1 \mathrm{~b}}$ entspricht. Dieser Generationenbruch findet sich auch in der Ukraine. 
ABB. 3

\section{Gerechtigkeitsurteile nach Bildungsgruppen im Vergleich*}

semipartielle $R^{2}$-Werte

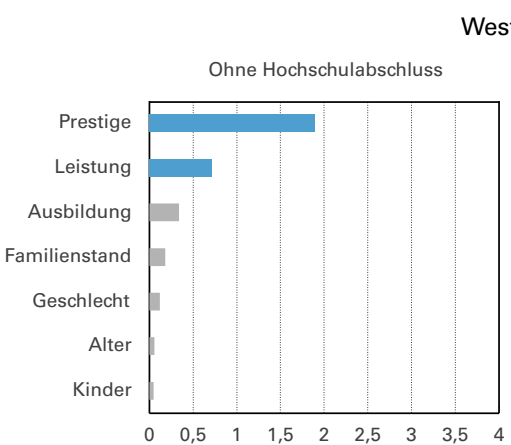

Westdeutschland
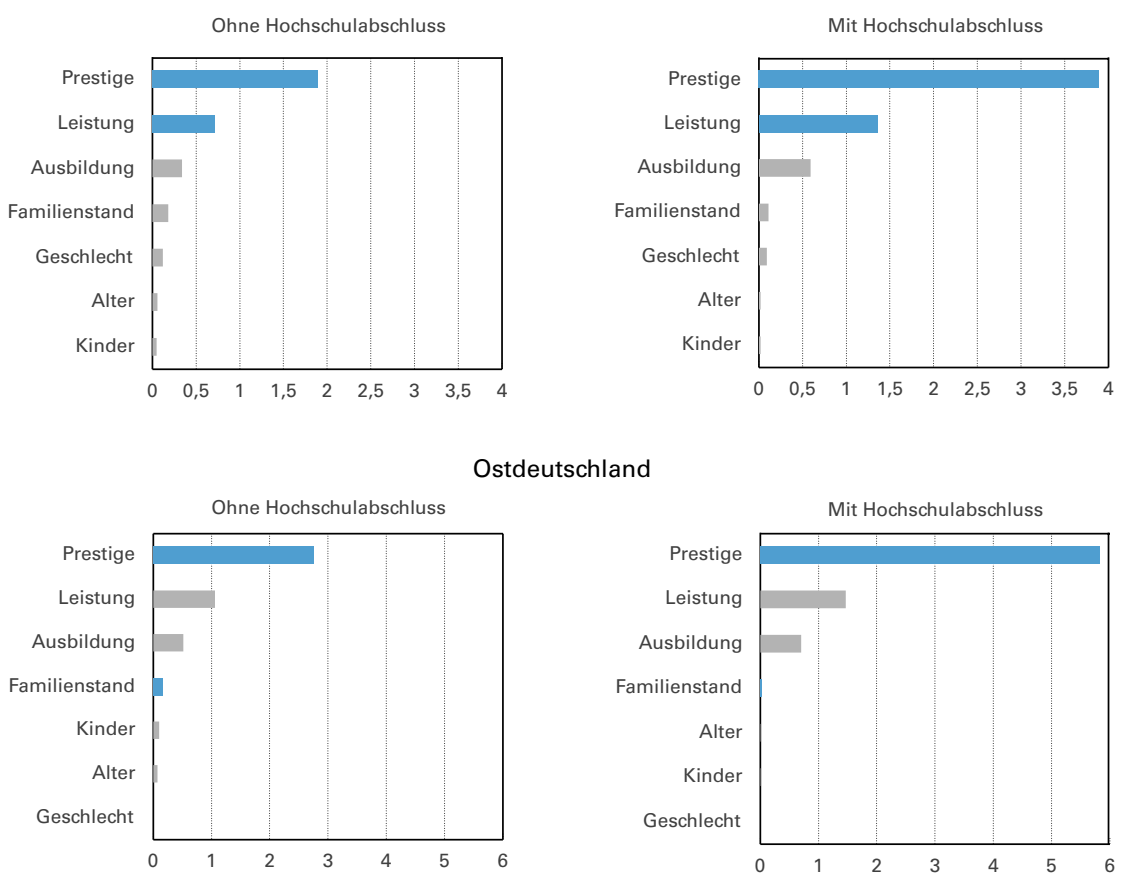

Ostdeutschland

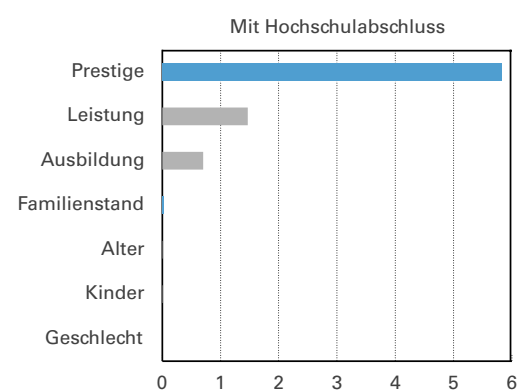

Ukraine

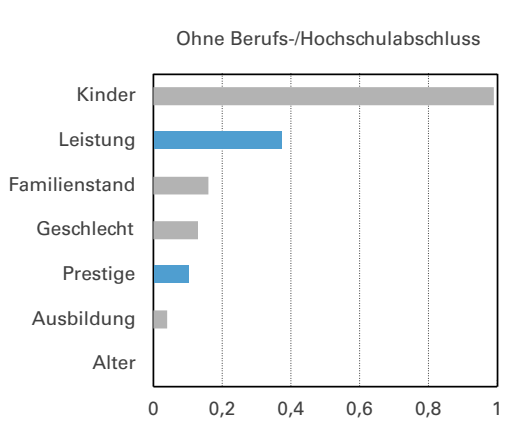

${ }^{*} \mathrm{~N}=15.072$ und 2.568 Vignettenurteile für die geringer und höher Gebildeten in Westdeutschland (628 und 107 Befragte); $\mathrm{N}=3.984$ und 984 Vignettenurteile (166 und 41 Befragte) für Ostdeutschland; $N=13.037$ und 8.476 Vignettenurteile für die geringer und höher Gebildeten in der Ukraine (1.087 und 707 Befragte). Für eine bessere Lesbarkeit sind die $x$-Achsen länderspezifisch formatiert.

Quelle: SOEP Pretest (2008) bzw. Omnibus (2009); Berechnungen der Autoren.

Dagegen ist der Familienstand, als ein Bestandteil von Bedarfsgerechtigkeit, in der jüngeren Generation weniger bedeutsam. Interessanterweise spielt die Ausbildung in der jüngeren Generation eine noch marginalere Rolle als für die ältere Befragtengruppe. Jüngere wie ältere Personen halten aber am Bedarfsprinzip „Kinder“ fest. Damit kann die Hypothese $\mathrm{H}_{1 \mathrm{~b}} \mathrm{zu}$ den Generationsunterschieden in den Transformationsländern teilweise bestätigt werden.

In Abbildung 3 sind die Analysen getrennt nach Bildungsgruppen dargestellt. Die Hypothese zum Eigeninteresse behauptet einen Zusammenhang der Bewertungsprin-

zipien mit der eigenen Statusposition $\left(\mathrm{H}_{3 \mathrm{a}}\right)$, welcher vor allem in stabilen Gesellschaften auftreten sollte $\left(\mathrm{H}_{3 \mathrm{~b}}\right)$ : Hat man selbst höhere Bildung und höheren Status, dann werden diese Kriterien auch stärker als gerechte Verteilungsprinzipien unterstützt. Für Westdeutschland kann dies eindeutig bestätigt werden: Bildungshöhere Personen greifen in stärkerem Ausmaß auf Prestige und Leistung (und zumindest tendenziell Ausbildung) als Kriterien zur Legitimation eines hohen Einkommens zurück als bildungsniedrigere Personen.

Auch in Ostdeutschland ist dies erkennbar: Dort unterscheidet sich der Einfluss des Prestiges statistisch signifikant zwischen den Bildungsgruppen. Personen mit Hochschulabschluss halten das Berufsprestige für die Legitimation von gerechtem Einkommen für deutlich relevanter als Personen ohne Hochschulabschluss. Personen mit niedrigem Bildungsniveau sind weiterhin eher geneigt, dem Familienstand eine zwar kleine, aber wahrnehmbare Rolle bei der Bewertung von gerechten Einkommen zuzugestehen. In der Ukraine unterscheiden sich wie in Westdeutschland die Bildungsgruppen im Hinblick auf die Honorierung von Leistung und Prestige. In beiden Bildungsgruppen behält aber das Bedarfskriterium „Kinder“ die größte relative Bedeutung. In allen Bevölkerungsgruppen gelten die Unterschiede zwischen den Bildungsgruppen übrigens auch innerhalb der jüngeren Altersgruppe (nicht dargestellte, aber auf Anfrage erhältliche Auswertungen). Dementsprechend handelt es sich bei den aufgedeckten Unterschieden nach Bildung nicht um einen verdeckten Alterseffekt. Insgesamt findet sich also Unterstützung für die Hypothesen $\mathrm{H}_{3 \mathrm{a}}$ und $\mathrm{H}_{3 \mathrm{~b}}$, wonach Gerechtigkeitsurteile speziell in stabilen Gesellschaften mit Eigeninteressen korrelieren.

\subsection{Vergleich der Gerechtigkeitsurteile mit realen Lohnschätzungen $\left(\mathrm{H}_{\mathrm{a}}, \mathrm{H} 2_{\mathrm{b}}\right)$}

Zur Beurteilung der beiden letzten noch offenen Hypothesen $\left(\mathrm{H}_{2 \mathrm{a}}, \mathrm{H}_{2 \mathrm{~b}}\right)$, wonach die von den Befragten herangezogenen Kriterien insbesondere in stabilen Gesellschaften mit den tatsächlichen Kriterien übereinstimmen (Adaption), muss die tatsächliche Bedeutung der Merkmale zunächst auf der Grundlage von geeigneten Datensätzen ermittelt werden. In Ergänzung zur eigenen Datenerhebung findet das SOEP 2008 (Wagner et al. 2007) für Analysen zu den beiden deutschen Landesteilen sowie der Ukrainian Longitudinal Monitoring Survey (ULMS) von 2004 für die Ukraine Verwendung (Kiev International Institute of Sociology 2004). ${ }^{\ominus}$ Für die Berechnungen werden die Stichproben und Operationalisierungen soweit wie möglich an diejenigen im FS-Modul angeglichen. Etwa werden für die deutschen

(9 Dieser Datensatz bietet die besten Vergleichswerte zum FSModul. Für den Datenzugang zum ULMS bedanken wir uns sehr herzlich beim Institute for the Study of Labor (IZA), Bonn. 


\section{Lohnschätzungen mit vorhandenen Datensätzen im Vergleich zu den Vignettenschätzungen*}

semipartielle $R^{2}$-Werte
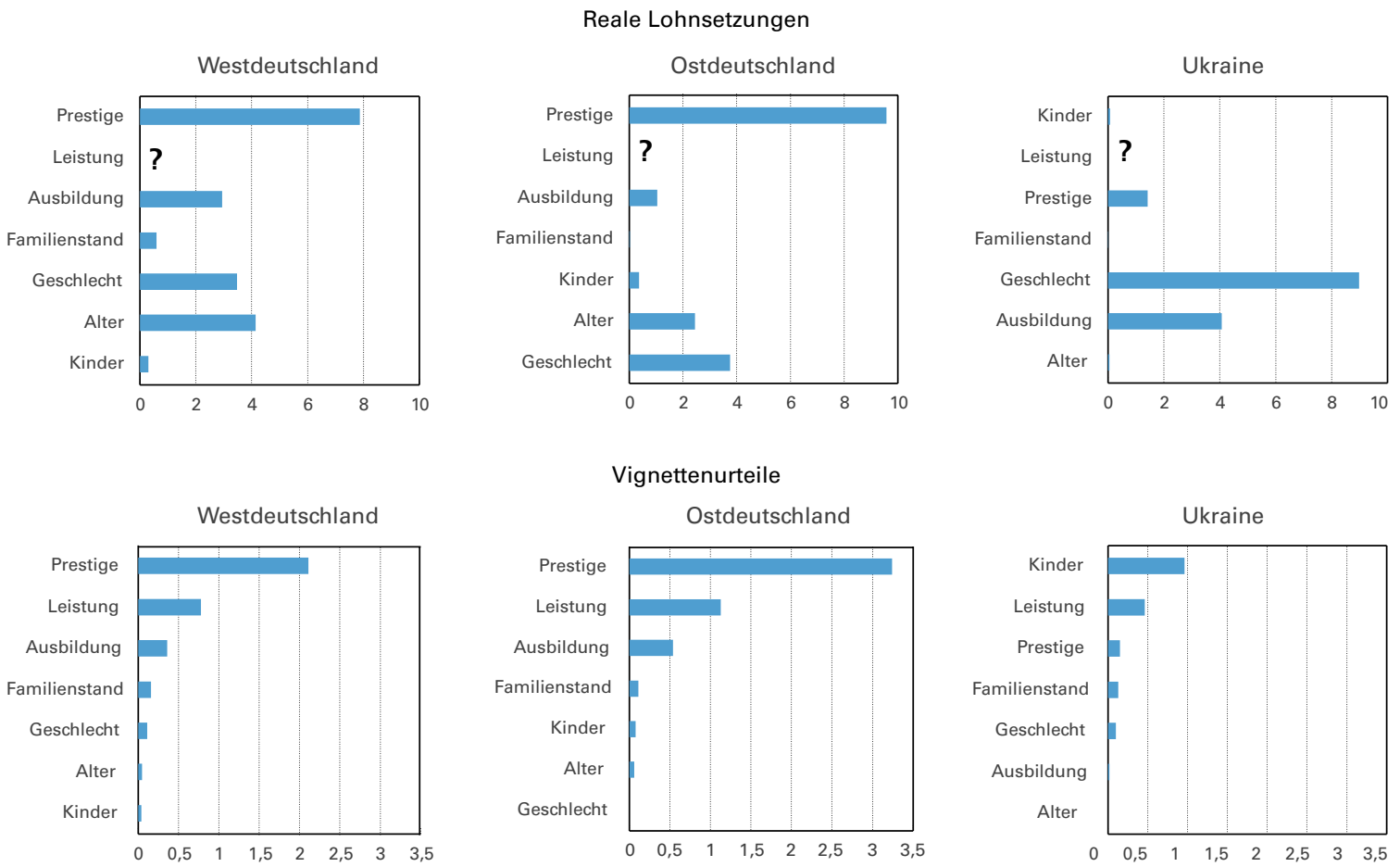

*Für die Einflussfaktoren auf die realen Gehälter: SOEP 2008 (eigene Berechnungen) mit N = 3.261 Befragten in Westdeutschland und 1.114 Befragten in Ostdeutschland sowie für die Ukraine ULMS 2004 (eigene Berechnungen) mit 1.233 Befragten. Es wurden jeweils nur abhängig Beschäftigte in Vollzeit und für das SOEP nur solche in Berufen mit Prestigewerten in einer Spannweite analog zu den Vignetten verwendet. Schätzungen der logarithmierten Bruttolöhne unter Kontrolle der Arbeitszeit. Für die Vignettenurteile: SOEP Pretest $2008 \mathrm{mit} \mathrm{N}=17.688$ Vignettenurteilen (737 Befragte) für Westdeutschland und 4.968 Vignettenurteilen (207 Befragte) für Ostdeutschland sowie Omnibus 2009 mit 21.549 Vignettenurteilen (1.797 Befragte) für die Ukraine.

Quelle: SOEP Pretest (2008) bzw. Omnibus (2009), ULMS (2004); Berechnungen der Autoren.

Landesteile nur Beschäftigte in Berufen analog zur Spannweite von Prestigewerten in dem FS-Modul des SOEP-Pretests berücksichtigt. Es werden ebenso nur analoge Altersund Bildungsgruppen und das Bruttoeinkommen herangezogen. Wiederum werden semipartielle $R^{2}$-Werte ausgewiesen (Abbildung 4; als abhängige Variable dienen in den Lohnschätzungen logarithmierte Bruttoeinkommen). $\mathrm{Zu}$ beachten ist, dass in den Lohnschätzungen keine Indikatoren für die Arbeitsleistung verwendet werden können.

Für den Vergleich der Lohnschätzung mit den Gerechtigkeitseinschätzungen zeigen sich in Westdeutschland einige Auffälligkeiten. Die Ausbildung von Erwerbspersonen ist ebenso wie das Geschlecht in den Lohnregressionen vergleichsweise bedeutsamer als in den Vignettenurteilen. Auch das Alter hat als Indikator für Berufserfahrung starken Einfluss auf die Lohnhöhe. In Ostdeutschland ist die Übereinstimmung der tatsächlichen Bestimmungsfaktoren von Lohnhöhen mit den Merkmalen, die gerechterweise den Lohn bestimmen sollten, recht hoch, wenn man von der Bedeutung des Geschlechts absieht. Insgesamt scheint es eine beachtliche, nicht nach Gruppen spezifische Orientie- rung der Gerechtigkeitseinschätzungen an den tatsächlichen Verhältnissen zu geben. In der Ukraine ist die Lohnbildung, wie sie sich aus den tatsächlichen Arbeitsmarktdaten schätzen lässt, sehr stark vom Geschlecht bestimmt. Beruflicher Erfolg, wie er sich in der Lohnhöhe ausdrückt, und der als gerecht empfundene Lohn sind in der Ukraine offensichtlich knapp 20 Jahre nach der Transformation nur lose verknüpft, zudem gelingt nur eine vergleichsweise geringe Aufklärung der tatsächlichen Lohnhöhe. ${ }^{(0)}$ Dies ist konform zur Annahme $\mathrm{H}_{2 \mathrm{~b}}$, dass ausgeprägte Adaptionen nach Transformationen erst mit einer gewissen Zeitverzögerung eintreten. Eine genaue Einschätzung der Adaptionsthese ist jedoch schwierig, weil die tatsächlichen Bestimmungsfaktoren der Lohnhöhe in der Ukraine unklar ausfallen und auch das tatsächliche verfügbare Einkommen weitaus weniger mit den gezahlten Bruttolöhnen aus abhängiger Beschäftigung

\footnotetext{
(10) Es wird ein $R^{2}$-Wert von 0,28 erreicht, während für die west- und ostdeutschen Lohnschätzungen $R^{2}$-Werte von 0,48 bzw. 0,46 erzielt werden.
} 


\section{Gerechte und reale Lohnabstände im Vergleich (West- und Ostdeutschland)*}
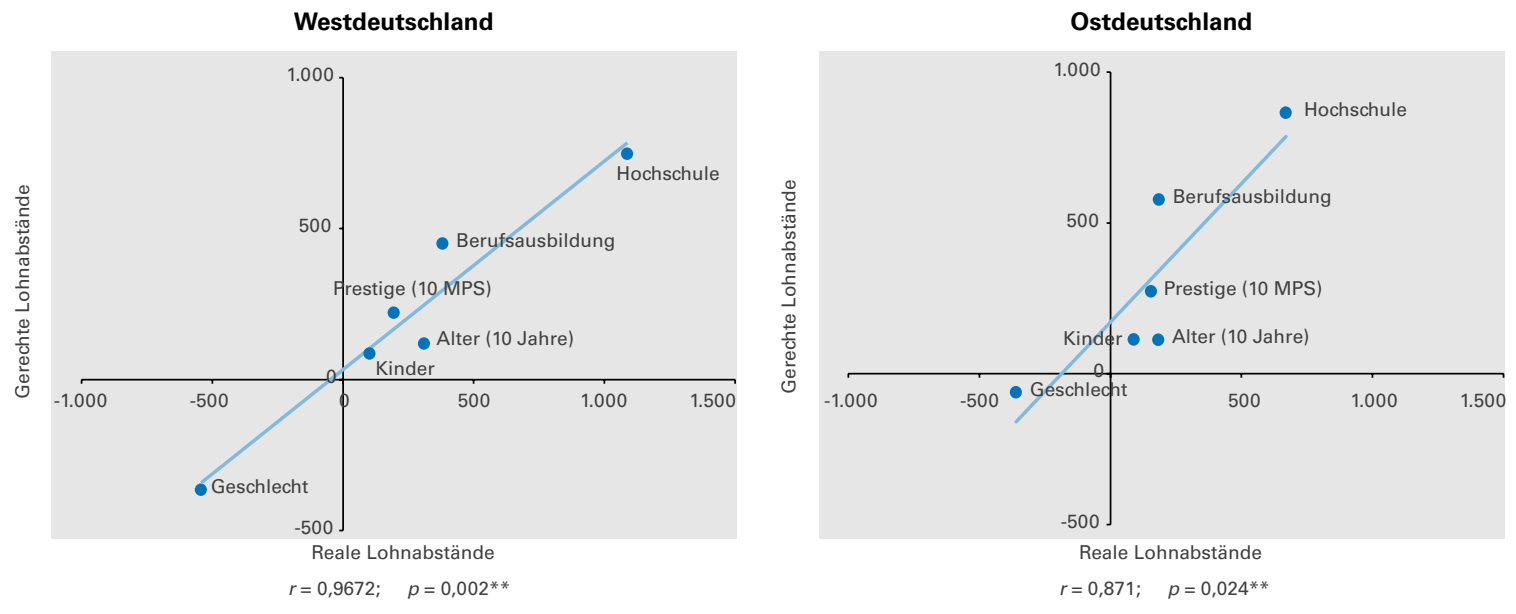

*Bei den Werten unter den Grafiken handelt es sich um die Korrelationskoeffizienten nach Pearson mit dazugehörigen Signifikanznieveaus. Signifikanzlegende: * $p<0,05 ; * * 0<0,01$.

Quelle: Für die verwendeten Datensätze, Fallzahlen und Kontrollvariablen siehe die Anmerkungen unter Abb. 4.

zusammenhängt. Nicht selten haben Menschen in der Ukraine beträchtliche Nebeneinkünfte aus einer zweiten Erwerbstätigkeit oder aus Schwarzarbeit (Round/Williams 2010). Zudem sind in den postsowjetischen Ländern Beziehungen (auch familiärer Art) für den beruflichen Erfolg sehr bedeutsam (Soroka/Zub 2008; Yakubovich/Kozina 2000; Yakubovich 2005). Zumindest für West- und Ostdeutschland gibt es jedoch Evidenz für die normative Kraft des Faktischen, wie es eindrücklich Abbildung 5 veranschaulicht. Auf Basis der erwähnten Formel von Jasso (2007) werden hier für die einzelnen Merkmale reale und gerechte Lohnabstände für die einzelnen Merkmale gegenübergestellt. In West- wie Ostdeutschland findet sich eine erstaunlich hohe Übereinstimmung zwischen den faktischen Verhältnissen und den Gerechtigkeitsurteilen aus der Befragungsstudie.

\section{Fazit und Diskussion}

Die empirische Untersuchung der Prinzipien von Einkom mensgerechtigkeit in Westdeutschland, Ostdeutschland und der Ukraine konnte in einem zentralen Aspekt die theoretische Erwartung nicht unterstützen: Beide Transformationsgesellschaften sind keineswegs weniger an Leistung als Gerechtigkeitsprinzip orientiert als der Vergleichsfall Westdeutschland. Die Überlegungen zur Bedeutung des Generationenbruchs in den Transformationsgesellschaften und zur Rolle des Eigeninteresses bei der Einschätzung von gerechten Entlohnungsgründen wurden hingegen bestätigt:
Jüngere Befragte unterstützen in beiden Transformationsgesellschaften im Vergleich zu älteren Personen stärker die Leistung (in Ostdeutschland und der Ukraine) und das Berufsprestige (in Ostdeutschland). Und die Befragten mit besseren Bildungsressourcen bewerten die Frage nach der normativen Bedeutung von Leistung und Prestige ebenfalls besonders positiv. Dies gilt vor allem in den stabileren Gesellschaften. Schließlich kann für West- und Ostdeutschland die normative Kraft des Faktischen belegt werden. In der Ukraine fallen dagegen die real messbare Entlohnung und die Gerechtigkeitsvorstellungen auseinander. Es herrscht dort vor allem eine starke normative Orientierung am Bedarfsprinzip (Kinder), wofür es in den zur Verfügung stehenden Daten zur realen Einkommenserzielung keine Entsprechung gibt.

Die Ukraine als post-sowjetische Transformationsgesellschaft weicht in einigen Aspekten deutlich von Ostdeutschland ab, nicht jedoch - wie bereits erwähnt - in der prinzipiellen Anerkennung von Leistung als Verteilungskriterium. Allerdings überrascht, wie stark in der Ukraine die Kinderzahl die Bewertung eines als gerecht empfundenen Bruttoeinkommens beeinflusst. Dieses Ergebnis verweist darauf, dass Arbeitgeber in der Ukraine als Patriarchen angesehen werden, die den Bedarf berücksichtigen sollten. Bereits erwähnt wurde das Problem der exakten Einkommensmessung in der Ukraine. Wegen des einfachen Steuersystems mit einem für alle einheitlichen Steuersatz von $15 \%$ ist hier zwar die Unterscheidung von Brutto- und Nettoeinkommen längst nicht so komplex wie in Deutschland, aber das Einkommen aus abhängiger Beschäftigung ist oftmals nur eine Quelle neben vielen anderen für das Haus- 
haltseinkommen. Schließlich ist überaus interessant, dass die Ausbildung im Meinungsbild der Befragten keine Rolle für die gerechte Lohnhöhe spielen sollte. Der Hintergrund hierfür ist vermutlich, dass das ukrainische Bildungssystem in den Augen der Befragten keine leistungsbezogenen $\mathrm{Ab}$ schlüsse vermittelt, weil an Universitäten Abschlussnoten und Doktortitel nicht selten nach Tarif verkauft werden (Heyneman et al. 2008; Osipian 2009). Auch die Berufe und ihr Prestige haben wenig Einkommenswert auf dem Arbeitsmarkt. Hierin liegen sicher große Hindernisse im weiteren Transformationspfad der Ukraine. In der jüngeren Generation gewinnt allerdings die individuelle Leistung im Vergleich zu Bedarfskriterien wie der Kinderzahl deutlich an Legitimationskraft.

Alles in allem zeigen sich also in unterschiedlichem Ausmaß Bestätigungen für die untersuchten Theorien. Die Gerechtigkeitseinschätzungen werden in sozialen Kontexten gelernt, sie werden nach individueller Nützlichkeit variiert und auch an bestehende Verhältnisse angepasst. Die starke Adaption könnte auch zur Erklärung von viel diskutierten Ergebnissen anderer Studien beitragen, etwa wenn höhere Einkommensgruppen ihre überproportionale Besteuerung als gerecht empfinden (Liebig/Mau 2005) und dies einfach als ein Hinweis auf gruppenbezogene Moral gedeutet wird - es könnte sich hierbei alternativ auch lediglich um eine Prägung durch die gegebenen Verhältnisse handeln. Die sehr hohe Entsprechung von Gerechtigkeitsurteilen und faktischen Verhältnissen ist vor allem aber angesichts der medialen (Zerr-)Bilder von unfähigen Bankern und Managern mit Millionengehältern überraschend. Sie erfordert eine stärkere theoretische Durchdringung. Zugleich sollte der Wandel von Gerechtig keitseinstellungen in Transformationsgesellschaften weiter beobachtet werden.

\section{LITERATUR}

Almond, G./Verba, S. (1963): The Civic Culture, Princeton

Alves, W./Rossi P. H. (1978): Who Should Get What? Fairness Judgments of the Distribution of Earnings, in: The American Journal of Sociology 84 (3), S. 541-564

Alwin, D. F. (1987): Distributive Justice and Satisfaction with Material WellBeing, in: American Sociological Review 52 (1), S. 83-95

Alwin, D. F./Krosnick, J. A. (1991): Aging, Cohorts and the Stability of Sociopolitical Orientations Over the Life Span, in: American Journal of Sociology 97 (1), S. 169-195

Arts, W./Hermkens, P./van Wijck, P. (1991): Income and the Idea of Justice: Principles, Judgments and their Framing, in: Journal of Economic Psychology 12 (1), S. $121-140$

Auspurg, K./Hinz, T./Liebig, S./Sauer, C. (2008): Wer verdient welches Einkommen? Ergebnisse eines Faktoriellen Surveys zur Einkommensgerechtigkeit in Deutschland, Universität Bielefeld/Universität Konstanz

Auspurg, K./Jäckle A. (2012): First Equals Most Important? Order Effects in Vignette-Based Measurement: University of Essex, Institute for Social \& Economic Research, ISER Working Paper 2012-01, Essex

Beck, M./Opp, K.-D. (2001): Der Faktorielle Survey und die Messung von Normen, in: Kölner Zeitschrift für Soziologie und Sozialpsychologie 53 (2), S. $283-306$

Bispinck, R./Dribbusch, H./Öz, F. (2008): Geschlechtsspezifische Lohndifferenzen nach dem Berufsstart und in der ersten Berufsphase. Eine Analyse von Einkommensdaten auf Basis der WSI-LohnSpiegel-Datenbank in Deutschland und im europäischen Vergleich: Hans-Böckler-Stiftung, Düsseldorf

Christoph, B. (2005): Zur Messung des Berufsprestiges. Aktualisierung der Magnitude-Prestigeskala auf die Berufsklassifikation ISCO88, ZUMA-Nachrichten (57), S. 79-127

Cohn, E. S./White, S. O./Sanders, J. (2000): Distributive and Procedural Justice in Seven Nations, in: Law and Human Behavior 24 (5), S. 553-579
Coleman, J. S. (1990): Foundations of SocialTheory, Cambridge/Mass. Festinger, L. (1957): A Theory of Cognitive Dissonance, Stanford, CA Hadler, M. (2005): Why Do People Accept Different Income Ratios? A Multi-level Comparison of Thirty Countries, in: Acta Sociologica 48 (2), S. 131-154 Heyneman, S. P./Anderson, K. H./Nuraliyeva, N. (2008): The Cost of Corruption in Higher Education, in: Comparative Education Review 52 (1) S. 1-25 Homans, G. C. (1973): Fundamental Social Processes, in: Smelser, N. J. (Hrsg.): Sociology: An Introduction, New York et al., S. 549-594

Homans, G. C. (1974): Social Behavior: Its Elementary Forms, New York Hox, J./Kreft, I. G./Hermkens, P. L. J. (1991): The Analysis of Factorial Surveys, in: Sociological Methods \& Research 19 (4), S. 493-510

Institute of Sociology of the National Academy of Sciences of Ukraine (2009): Ukrajins'ke suspil'stvo 1992-2009. Dynamika social'nych zmin. Kyjiv: IS NANU, Azbuka [Ukrainian Society in 1992-2009. Dynamics of Social Changes. Kyiv]

Jann, B. (2005): Einführung in die Statistik, München

Jasso, G. (2006): Factorial-Survey Methods for Studying Beliefs and Judgments, in: Sociological Methods and Research 34 (3), S. 334-423

Jasso, G. (2007): Studying Justice: Measurement, Estimation, and Analysis of the Actual Reward and the Just Reward: Institute for the Study of Labor (IZA), IZA Discussion Paper (2592), Bonn

Jasso, G./Rossi, P. H. (1977): Distributive Justice and Earned Income, in: American Sociological Review 42 (4), S. 639-651

Jasso, G./Webster, M. (1999): Assessing the Gender Gap in Just Earnings and Its Underlying Mechanisms, in: Social Psychology Quarterly 62 (4),

S. $367-380$

Kiev International Institute of Sociology (2004): Ukrainian Longitudinal Monitoring Survey 2004, Technical Report, Kiev, http://idsc.izaorg/?page=86\&wid= 622 (letzter Zugriff: 20.06.2012)

Kluegel, J. R./Mason, D. S./Wegener, B. (Hrsg.) (1995): Social Justice and Political Change. Public Opinion in Capitalist and Post-Communist States, Berlin/ New York

Liebig, S. (1997): Soziale Gerechtigkeitsforschung und Gerechtigkeit in Unternehmen, München/Mering

Liebig S./Lengfeld, H./Mau, S. (2004): Einleitung: Gesellschaftliche Verteilungsprobleme und der Beitrag der soziologischen Gerechtigkeitsforschung, in: Dies. (Hrsg.): Verteilungsprobleme und Gerechtigkeit in modernen Gesellschaften, Frankfurt a. M., S. 7-28

Liebig S./Mau, S. (2005): Wann ist ein Steuersystem gerecht? Einstellungen zu allgemeinen Prinzipien der Besteuerung und zur Gerechtigkeit der eigenen Steuerlast, in: Zeitschrift für Soziologie 34 (6), S. 468-491

Liebig, S./Wegener, B. (1995): Primäre und sekundäre Ideologien: Ein Vergleich von Gerechtigkeitsvorstellungen in Deutschland und den USA, in: Müller, H.-P./Wegener, B. (Hrsg.): Soziale Ungleichheit und soziale Gerechtigkeit, Opladen, S. 265-293

Lindenberg, S. (1989): Social Production Functions, Deficits, and Social Revolutions, in: Rationality and Society 1 (1), S. 51-77

Marshall, G./Swift, A./Routh, D./Burgoyne, C. (1999): What Is and What Ought to Be. Popular Beliefs about Distributive Justice in Thirteen Countries, in: European Sociological Review 15 (4), S. 349-367

Mason, D. (1995): Justice, Socialism, and Participation in the Postcommunist States, in: Kluegel, J. R./Mason, D./Wegener, B. (Hrsg.): Social Justice and Political Change. Public Opinion in Capitalist and Post-Communist States, Berlin/ New York, S. 49-80

Merton, R. K. (1938): Social Structure and Anomie, in: American Sociological Review 3 (5), S. 672-682

Merton, R. K. (1957): SocialTheory and Social Structure, Glencoe/IL.

Miller, D. (2008): Grundsätze sozialer Gerechtigkeit, Frankfurt a. M.

Oerter, R./Montada, L. (1998): Entwicklungspsychologie, Weinheim

Oksamytna, S./Patrakova, A. (2007): Ijerarchija prestyžnosti profesij i zanjat' [The hierarchy of professions and occupational prestige], Ukrajins'ke suspil'stvo, Kyjiv.: IS NANU, S. 170-179

Opp, K.-D. (1983): Die Entstehung sozialer Normen, Tübingen

Opp, K.-D. (1988): Grievances and Participation in Social Movements, in: American Sociological Review 53 (6), S. 853-864

Osipian, A. L. (2009): Corruption and Reform in Higher Education in Ukraine, in: Canadian and International Education 38 (2), S. 104-122

Pickel, G./Pollack, D./Müller, O./Jacobs, J. (2006): Osteuropas Bevölkerung auf dem Weg in die Demokratie. Repräsentative Untersuchungen in Ostdeutschland und zehn osteuropäischen Transformationsstaaten, Wiesbaden Ridgeway, C. (1991): The Social Construction of Status Value: Gender and Other Nominal Characteristics, in: Social Forces 70 (2), S. 367-386 
Rogers, W. H. (1993): Regression Standard Errors in Clustered Samples, in Stata Technical Bulletin 13 (3), S. 19-23

Rossi, P. H./Nock, S. L. (Hrsg.) (1982): Measuring Social Judgments. The Factorial Survey Approach, Beverly Hills et al.

Round, J./Williams, C. (2010): Coping with the Social Costs of 'Transition': Everyday Life in Post-Soviet Russia and Ukraine, in: European Urban and Regional Studies 17 (2), S. 183-196

Sauer, C./Liebig, S./Auspurg, K./Hinz, T./Donaubauer, A./Schupp, J. (2009): A Factorial Survey on the Justice of Earnings Within the SOEP-Pretest 2008: Institute for the Study of Labor (IZA), IZA Discussion Paper (4663), Bonn Soofi, E. S./Retzer, J. J./Yasai-Ardekani, M. (2000): A Framework for Measuring the Importance of Variables with Applications to Management Research and Decision Models, in: Decision Sciences 31 (3), S. 595-625

Soroka, J./Zub, T. (2008): „Vidmova vid bahatstva” ta zminy u spryjnjatti social'noji nerivnosti. [„Denial of wealth" and changes in perception of social inequality], in: Sociolohija: teorija, metody, marketynh (2008/1), S. 222-236 Statistisches Bundesamt (2009): Verdienste und Arbeitskosten. Verdienststrukturerhebung 2006 - Verdienste nach Berufen, Statistisches Bundesamt, Wiesbaden

Wagner, G. G./Frick, J. R./Schupp, J. (2007): The German Socio-Economic Panel Study (SOEP) - Scope, Evolution and Enhancements, in: Schmollers Jahrbuch 127 (1), S. 161-191

Walster, E./Berscheid, E./Walster, W. G. (1973): New Directions in Equity Research, in: Journal of Personality and Social Psychology 25 (2), S. 151-176 Wegener, B./Liebig, S. (1993): Eine Grid-Group-Analyse sozialer Gerechtigkeit: Die neuen und alten Bundesländer im Vergleich, in: Kölner Zeitschrift für Soziologie und Sozialpsychologie 45 (4), S. 668-690

Wegener, B./Liebig, S. (1995): Hierarchical and Social Closure Conceptions of Distributive Social Justice: A Comparison of East and West Germany, in: Kluegel, J. R./Mason, D. S./Wegener, B. (Hrsg): Social Justice and Political Change. Public Opinion in Capitalist and Post-Communist States, Berlin/New York,

S. $263-284$

Wegener, B./Lippl, B./Christoph, B. (2000): Justice Ideologies, Perceptions of Reward Justice, and Transformation: East and West Germany in Comparison, in: Mason, D. S./Kluegel, J. R. (Hrsg.): Marketing Democracy. Changing Opinion About Inequality and Politics in East Central Europe, S. 122-160

Wegener, B./Steinmann, S. (1995): Justice Psychophysics in the Real World: Comparing Income Justice and Income Satisfaction in East and West Germany, in: Kluegel, J. R./Mason, D. S./Wegener, B. (Hrsg): Social Justice and Politi cal Change. Public Opinion in Capitalist and Post-Communist States, Berlin/ New York, S. 151-175
Wooldridge, J. M. (2006): Introductory Econometrics: A Modern Approach, Mason et al.

Yakubovich, V. (2005): WeakTies, Information, and Influence: How Workers Find Jobs in a Local Russian Labor Market, in: American Sociological Review 70 (3), S. 408-421

Yakubovich, V./Kozina, I. (2000): The Changing Significance of Ties: an Exploration of the Hiring Channels in the Russian Transitional Labor Market, in: International Sociology 15 (3), S. 479-500

\section{AUTOREN}

KATRIN AUSPURG, Dr., Soziologin, ist Wissenschaftliche Mitarbeiterin an der Universität Konstanz. Arbeitsschwerpunkte: Methoden der empirischen Sozialforschung, Familiensoziologie, soziologischeTheorie.

katrin.auspurg@uni-konstanz.de

KSENIJA GATSKOVA, M.A., Soziologin, Doktorandin im Fach Soziologie der Universität Konstanz und wissenschaftliche Mitarbeiterin am Institut für Ostund Südosteuropaforschung, Regensburg. Arbeitsschwerpunkte: Gerechtigkeitsforschung, Osteuropa.

gatskova@googlemail.com

THOMAS HINZ, Prof. Dr., Soziologe, lehrt und forscht an der Universität Konstanz. Arbeitsschwerpunkte: Surveyforschung, Arbeitsmarkt- und Wirtschaftssoziologie, Bildungssoziologie.

thomas.hinz@uni-konstanz.de 